FACTA UNIVERSITATIS (NIŠ)

Ser. Math. Inform. Vol. 36, No 1 (2021), 79-88

https://doi.org/10.22190/FUMI200310007K

Original Scientific Paper

\title{
A NEW GENERALIZATION OF $M$-METRIC SPACE WITH SOME FIXED POINT THEOREMS
}

\author{
Erdal Karapınar ${ }^{1}$, Kushal Roy ${ }^{2}$ and Mantu Saha ${ }^{2}$ \\ ${ }^{1}$ Department of Mathematics, Çankaya University, 06790, Etimesgut, Ankara, Turkey \\ ${ }^{2}$ Department of Mathematics, The University of Burdwan, \\ Purba Bardhaman-713104, West Bengal, India
}

\begin{abstract}
In this paper, we introduce a new sequential space as a generalization of $M$-metric spaces and $M_{b}$-metric spaces. In this generalized space we define two contractive mappings namely $\mathfrak{m}$-contraction and $\mathfrak{m}$-quasi-contraction and prove some fixed point theorems for such type of mappings. Several illustrative examples have been presented in strengthening the hypothesis of our theorems.

Keywords: M-metric space, Fixed point theory, stability
\end{abstract}

\section{Introduction and Preliminaries}

The notion of metric has been generalized in several direction, see e.g. $[1,2,6,7$, 8, 9]. Among all, we focus on partial metric and $M$-metric. The concept of partial metric space was first introduced by S. Matthews [1] in 1994 as a generalization of usual metric spaces. If $(X, p)$ is a partial metric space then $p(\mu, \mu), \mu \in X$ need not to be zero. Partial metric spaces have vast application potential, in particular, it has been used in the construction of the topological structures in the study of information science, computer science, etc. In 2014, Asadi et al. [2] have extended the notion of the partial metric space: $M$-metric space. The authors [2] proved the Banach contraction principle in the context of the complete $M$-metric space. The definition of $M$-metric is given as follows:

Throughout the manuscript all considered sets are nonempty. Further, the notation $X^{2}$ denotes the cross-product of the set $X$, that is, $X^{2}: X \times X$.

Received March 10, 2020; accepted January 9, 2021.

Corresponding Author: Erdal Karapınar, Department of Mathematics, Çankaya University, 06790, Etimesgut, Ankara, Turkey | E-mail: erdal.karapinar@cankaya.edu.tr

2010 Mathematics Subject Classification. Primary 47H10; Secondary 54H25, 54E50.

C 2021 by University of Niš, Serbia | Creative Commons License: CC BY-NC-ND 
Definition 1.1. Let $m: X^{2} \rightarrow[0, \infty)$ be a function over a set $X$. Then $(X, m)$ is said to be an $M$-metric space if $m$ satisfies the following conditions:

(m1) $m(p, p)=m(q, q)=m(p, q)$ if and only if $p=q$;

(m2) $m_{p q} \leq m(p, q)$

(m3) $m(p, q)=m(q, p)$;

$(\mathrm{m} 4) m(p, q)-m_{p q} \leq\left(m(p, r)-m_{p r}\right)+\left(m(r, q)-m_{r q}\right)$ for all $p, q, r \in X$,

where

and

$$
m_{p q}=\min \{m(p, p), m(q, q)\}
$$

$$
M_{p q}=\max \{m(p, p), m(q, q)\} .
$$

It is seen that any partial metric space is an $M$-metric space. In [2] authors have presented an example of $M$-metric that does not form a partial metric.

Example 1.1. Let $X=\{1,2,3\}$ and $m: X^{2} \rightarrow[0, \infty)$ be defined by $m(1,1)=1$, $m(2,2)=9, m(3,3)=5$ and

In 2015, Jleli-Samet [5] introduce a new generalization of the notion of metric spaces that involves $b$-metric and standard metric. Inspired by this work, we characterize the $M$-metric space and observed a new metric space. We present an example to indicate the novelty of this notion. Further, we observe some fixed point results in the setting of this new $M$-metric space.

\section{Main results}

In this section we introduce a generalized $M$-metric space namely $m^{*}$-metric space, as follows:

Let $\mathfrak{m}: X^{2} \rightarrow[0, \infty)$ be a function such that $\mathfrak{m}_{p q}=\min \{\mathfrak{m}(p, p), \mathfrak{m}(q, q)\}$ and $\mathcal{M}_{p q}=\max \{\mathfrak{m}(p, p), \mathfrak{m}(q, q)\}$. Let us define the set

$$
M(\mathfrak{m}, X, p)=\left\{\left\{p_{n}\right\} \subset X: \lim _{n \rightarrow \infty}\left(\mathfrak{m}\left(p_{n}, p\right)-\mathfrak{m}_{p_{n} p}\right)=0\right\}
$$

for all $p \in X$.

Definition 2.1. A function $\mathfrak{m}: X \times X \rightarrow[0, \infty)$, over a set $X$, is called an $m^{*}-$ metric if the following conditions hold:

$(\mathfrak{m} 1) \mathfrak{m}(p, p)=\mathfrak{m}(q, q)=\mathfrak{m}(p, q)$ if and only if $p=q, p, q \in X$;

$(\mathfrak{m} 2) \mathfrak{m}_{p q} \leq \mathfrak{m}(p, q)$ for all $p, q \in X$;

$(\mathfrak{m} 3) \mathfrak{m}(p, q)=\mathfrak{m}(q, p)$ for all $p, q \in X$;

$(\mathfrak{m} 4)$ there exists some $b>0$ such that for any $(p, q) \in X^{2}$ and $\left\{p_{n}\right\} \in$ $M(\mathfrak{m}, X, p)$ we have

$$
\mathfrak{m}(p, q)-\mathfrak{m}_{p q} \leq b \limsup _{n \rightarrow \infty}\left(\mathfrak{m}\left(p_{n}, q\right)-\mathfrak{m}_{p_{n} q}\right) .
$$

The pair $(X, \mathfrak{m})$ is called an $m^{*}$-metric space. 
In the following example we show that the newly defined $m^{*}$-metric space is more stronger than $M$-metric space.

Example 2.1. Let $X=\mathbb{N}$ and we define $\mathfrak{m}: X^{2} \rightarrow \mathbb{R}_{+}$by $\mathfrak{m}(n, n)=1$ for all $n \in \mathbb{N}$, $\mathfrak{m}(1,2)=\mathfrak{m}(2,1)=4, \mathfrak{m}(1, n)=\mathfrak{m}(n, 1)=1+\frac{1}{n}$ for all $n \geq 3, \mathfrak{m}(n, 2)=\mathfrak{m}(2, n)=\frac{5}{2}$ for all $n \geq 3$ and $\mathfrak{m}(n, k)=\mathfrak{m}(k, n)=3$ for any $n, k \notin\{1,2\}$. Here $M(\mathfrak{m}, X, 1)=$ $\{\{1,1, \ldots\},\{3,4,5, \ldots\}\}$ and for any other $x \in \mathbb{N}, M(\mathfrak{m}, X, x)$ contains only the constant sequence $\{x, x, \ldots\}$. Then one can easily check that $(X, \mathfrak{m})$ is an $m^{*}$-metric space.

Remark 2.1. In 2016, Mlaiki [10] defined $M_{b}$-metric space, by replacing the axiom (m4) in Definition 1.1 by

$$
\left(m_{b} 4\right) m_{b}(p, q)-m_{b_{p q}} \leq s\left[\left(m_{b}(p, r)-m_{b_{p r}}\right)+\left(m_{b}(r, q)-m_{b_{r q}}\right)\right] .
$$

Now we show that $(X, \mathfrak{m})$ in Example 2.1 is not an $M_{b}$-metric space for any $b>0$. Here we see that $\mathfrak{m}(n, k)-\mathfrak{m}_{n k}=2$ for any $n, k \geq 3$. But $b\left[\left(\mathfrak{m}(n, 1)-\mathfrak{m}_{n 1}\right)+\left(\mathfrak{m}(1, k)-\mathfrak{m}_{1 k}\right)\right]=$ $b\left[\frac{1}{n}+\frac{1}{k}\right] \rightarrow 0$ as $n, k \rightarrow \infty$ for any $b>0$. This proves our assertion.

Remark 2.2. (1) Let $(X, m)$ be an $M$-metric space (See Definition 1.1). Clearly $m$ satisfies the conditions $(\mathfrak{m} 1),(\mathfrak{m} 2)$ and $(\mathfrak{m} 3)$. Let $(p, q) \in X^{2}$ and $\left\{p_{n}\right\} \subset X$ be such that $\lim _{n \rightarrow \infty}\left(m\left(p_{n}, p\right)-m_{p_{n} p}\right)=0$ then from condition $(m 4)$ we have

$$
m(p, q)-m_{p q} \leq\left(m\left(p, p_{n}\right)-m_{p p_{n}}\right)+\left(m\left(p_{n}, q\right)-m_{p_{n} q}\right)
$$

for all $n \in \mathbb{N}$. Taking $n \rightarrow \infty$ we can easily see that $m$ satisfies the condition $(\mathfrak{m} 4)$. Hence $m$ satisfies all the conditions of $m^{*}$-metric and therefore $(X, m)$ is an $m^{*}$-metric space.

(2) Let $\left(X, m_{b}\right)$ be an $M_{b}$-metric space with coefficient $s \geq 1$. Then it is clear that $m_{b}$ satisfies the conditions ( $\left.\mathfrak{m} 1\right),(\mathfrak{m} 2)$ and $(\mathfrak{m} 3)$. Let $(p, q) \in X^{2}$ and $\left\{p_{n}\right\} \subset X$ be such that $\lim _{n \rightarrow \infty}\left(m_{b}\left(p_{n}, p\right)-m_{b_{p_{n}}}\right)=0$ then from condition $\left(m_{b} 4\right)$ we have

$$
m_{b}(p, q)-m_{b_{p q}} \leq s\left[\left(m_{b}\left(p, p_{n}\right)-m_{b_{p p_{n}}}\right)+\left(m_{b}\left(p_{n}, q\right)-m_{b_{p_{n} q}}\right)\right]
$$

for all $n \in \mathbb{N}$. Taking $n \rightarrow \infty$ it can be easily seen that $m_{b}$ satisfies the condition ( $\left.\mathfrak{m} 4\right)$. Hence $m_{b}$ satisfies all the conditions of $m^{*}$-metric and therefore $\left(X, m_{b}\right)$ is an $m^{*}$-metric space.

Definition 2.2. Let $(X, \mathfrak{m})$ be an $m^{*}$-metric space.

(1) A sequence $\left\{p_{n}\right\} \subset X$ is said to be convergent to an element $p \in X$ if $\lim _{n \rightarrow \infty}\left(\mathfrak{m}\left(p_{n}, p\right)-\mathfrak{m}_{p_{n} p}\right)=0$ i.e. $\left\{p_{n}\right\} \in M(\mathfrak{m}, X, p)$.

(2) A sequence $\left\{p_{n}\right\} \subset X$ is said to be Cauchy if $\lim _{n, k \rightarrow \infty}\left(\mathfrak{m}\left(p_{n}, p_{k}\right)-\mathfrak{m}_{p_{n} p_{k}}\right)$ and $\lim _{n, k \rightarrow \infty}\left(\mathcal{M}_{p_{n} p_{k}}-\mathfrak{m}_{p_{n} p_{k}}\right)$ exist and finite.

(3) A sequence $\left\{p_{n}\right\} \subset X$ is said to be 0 -Cauchy if $\lim _{n, k \rightarrow \infty}\left(\mathfrak{m}\left(p_{n}, p_{k}\right)-\mathfrak{m}_{p_{n} p_{k}}\right)=0$ and $\lim _{n, k \rightarrow \infty}\left(\mathcal{M}_{p_{n} p_{k}}-\mathfrak{m}_{p_{n} p_{k}}\right)=0$.

(4) An $m^{*}$-metric space $(X, \mathfrak{m})$ is said to be complete if every Cauchy sequence $\left\{p_{n}\right\} \subset X$ is convergent to some point $z \in X$ with $\lim _{n \rightarrow \infty}\left(\mathcal{M}_{p_{n} z}-\mathfrak{m}_{p_{n} z}\right)=0$. 
Definition 2.3. Let $(X, \mathfrak{m})$ be an $m^{*}$-metric space and $T: X \rightarrow X$ be a mapping. Then $T$ is said to be continuous at $\varsigma \in X$ if $\left\{p_{n}\right\} \in M(\mathfrak{m}, X, \varsigma)$ implies $\left\{T p_{n}\right\} \in$ $M(\mathfrak{m}, X, T \varsigma)$.

Proposition 2.1. Let $(X, \mathfrak{m})$ be an $m^{*}-$ metric space and $p, q \in X$. If $\left\{p_{n}\right\} \in$ $M(\mathfrak{m}, X, p) \cap M(\mathfrak{m}, X, q)$ then $\mathfrak{m}(p, q)=\mathfrak{m}_{p q}$. Moreover if $\mathfrak{m}(p, p)=\mathfrak{m}(q, q)$ then $p=q$.

Proof. Since $\left\{p_{n}\right\} \in M(\mathfrak{m}, X, p) \cap M(\mathfrak{m}, X, q)$, we have

$$
\mathfrak{m}(p, q)-\mathfrak{m}_{p q} \leq b \limsup _{n \rightarrow \infty}\left(\mathfrak{m}\left(p_{n}, q\right)-\mathfrak{m}_{p_{n} q}\right)=0
$$

implying that $\mathfrak{m}(p, q)-\mathfrak{m}_{p q}=0$ that is $\mathfrak{m}(p, q)=\mathfrak{m}_{p q}$. If $\mathfrak{m}(p, p)=\mathfrak{m}(q, q)$ also, then clearly $p=q$.

Proposition 2.2. Let $\left\{p_{n}\right\}$ be a 0 -Cauchy sequence in an $m^{*}-$ metric space $(X, \mathfrak{m})$. If $\left\{p_{n}\right\}$ has a convergent subsequence $\left\{p_{n_{k}}\right\}$ such that $\left\{p_{n_{k}}\right\} \in M(\mathfrak{m}, X, z)$ then $\left\{p_{n}\right\}$ is also convergent to $z \in X$.

Proof. Since $\left\{p_{n}\right\}$ is 0-Cauchy we have $\lim _{n, k \rightarrow \infty}\left(\mathfrak{m}\left(p_{n}, p_{k}\right)-\mathfrak{m}_{p_{n} p_{k}}\right)=0$ and $\lim _{n, k \rightarrow \infty}\left(\mathcal{M}_{p_{n} p_{k}}-\mathfrak{m}_{p_{n} p_{k}}\right)=0$. Also it is given that $\lim _{k \rightarrow \infty}\left(\mathfrak{m}\left(p_{n_{k}}, z\right)-\mathfrak{m}_{p_{n_{k}} z}\right)=0$. Now,

$$
\mathfrak{m}\left(p_{p}, z\right)-\mathfrak{m}_{p_{p} z} \leq b \limsup _{k \rightarrow \infty}\left(\mathfrak{m}\left(p_{p}, p_{n_{k}}\right)-\mathfrak{m}_{p_{p} p_{n_{k}}}\right)
$$

for all $p \in \mathbb{N}$. Which implies that

$$
\lim _{p \rightarrow \infty}\left[\mathfrak{m}\left(p_{p}, z\right)-\mathfrak{m}_{p_{p}} z\right] \leq b \lim _{p \rightarrow \infty} \limsup _{k \rightarrow \infty}\left(\mathfrak{m}\left(p_{p}, p_{n_{k}}\right)-\mathfrak{m}_{p_{p} p_{n_{k}}}\right)=0 .
$$

Therefore $\lim _{p \rightarrow \infty}\left(\mathfrak{m}\left(p_{p}, z\right)-\mathfrak{m}_{p_{p} z}\right)=0$, implying that $\left\{p_{n}\right\}$ is convergent to $z$.

\section{Topological $m^{*}$-metric space}

Definition 3.1. Let $(X, \mathfrak{m})$ be an $m^{*}$-metric space. The open and closed ball of center at $p \in X$ and radius $t>0$ in $X$ are defined as follows:

$$
\begin{aligned}
B^{\mathfrak{m}}(p, t) & =\left\{q \in X: \mathfrak{m}(p, q)<\mathfrak{m}_{p q}+t\right\} \\
B^{\mathfrak{m}}[p, t] & =\left\{q \in X: \mathfrak{m}(p, q) \leq \mathfrak{m}_{p q}+t\right\}
\end{aligned}
$$

Remark 3.1. One can easily check that the collection

$$
\tau_{\mathfrak{m}}=\varnothing \cup\left\{U(\neq \varnothing) \subset X: \text { for any } p \in U \text { there exists } t>0 \text { such that } B^{\mathfrak{m}}(p, t) \subset U\right\},
$$

forms a topology on $X$.

Definition 3.2. Let $(X, \mathfrak{m})$ be an $m^{*}$-metric space and $\Delta \subset X$. Then $\Delta$ is said to be closed if there exists an open set $U \subset X$ such that $\Delta=U^{c}$. 
Proposition 3.1. Let $(X, \mathfrak{m})$ be an $m^{*}$-metric space and $\Delta \subset X$ be closed. Let $\left\{p_{n}\right\} \subset \Delta$ be such that $\left\{p_{n}\right\} \in M(\mathfrak{m}, X, z)$, then $z \in \Delta$.

Proof. If possible let $z \notin \Delta$. Then $z \in \Delta^{c}=U$, where $U$ is open. So there exists $t>0$ such that $B^{\mathfrak{m}}(z, t) \subset U$. Now $\lim _{n \rightarrow \infty}\left(\mathfrak{m}\left(p_{n}, z\right)-\mathfrak{m}_{p_{n} z}\right)=0$ so for $t>0$ there exists $N \in \mathbb{N}$ such that $\mathfrak{m}\left(p_{n}, z\right)-\mathfrak{m}_{p_{n} z}<t$ whenever $n \geq N$. Thus $p_{n} \in B^{\mathfrak{m}}(z, t) \subset U$ for all $n \geq N$, a contradiction. Hence $z \in \Delta$.

Definition 3.3. Let $(X, \mathfrak{m})$ be an $m^{*}-$ metric space and $B \subset X$. Then $\operatorname{diam}(B)=$ $\sup \left\{\max \left\{\mathfrak{m}(p, q)-\mathfrak{m}_{p q}, \mathcal{M}_{p q}-\mathfrak{m}_{p q}\right\}: p, q \in B\right\}$.

Definition 3.4. In an $m^{*}$-metric space $(X, \mathfrak{m})$, a sequence $\left\{\Delta_{n}\right\}$ of subsets of $X$ is said to be decreasing if $\Delta_{1} \supset \Delta_{2} \supset \Delta_{3} \supset \ldots$.

Theorem 3.1. Let $(X, \mathfrak{m})$ be a complete $m^{*}$-metric space and $\left\{\Delta_{n}\right\}$ be a decreasing sequence of nonempty closed subsets of $X$ such that diam $\left(\Delta_{n}\right) \rightarrow 0$ as $n \rightarrow \infty$. Then the intersection $\cap_{n=1}^{\infty} \Delta_{n}$ contains exactly one point.

Proof. Let $p_{n} \in \Delta_{n}$ be arbitrary for all $n \in \mathbb{N}$. Since $\left\{\Delta_{n}\right\}$ is decreasing, we have $\left\{p_{n}, p_{n+1}, \ldots\right\} \subset \Delta_{n}$ for all $n \in \mathbb{N}$.

Now for any $n, p \in \mathbb{N}$ with $n, p \geq k$ we have $\max \left\{\mathfrak{m}\left(p_{n}, p_{p}\right)-\mathfrak{m}_{p_{n} p_{p}}, \mathcal{M}_{p_{n} p_{p}}-\right.$ $\left.\mathfrak{m}_{p_{n} p_{p}}\right\} \leq \operatorname{diam}\left(\Delta_{k}\right), k \geq 1$. Let $\epsilon>0$ be given. Then there exists some $q \in \mathbb{N}$ such that $\operatorname{diam}\left(\Delta_{q}\right)<\epsilon$ since $\operatorname{diam}\left(\Delta_{n}\right) \rightarrow 0$ as $n \rightarrow \infty$. From this it follows that $\max \left\{\mathfrak{m}\left(p_{n}, p_{p}\right)-\mathfrak{m}_{p_{n} p_{p}}, \mathcal{M}_{p_{n} p_{p}}-\mathfrak{m}_{p_{n} p_{p}}\right\}<\epsilon$ whenever $n, p \geq q$. Therefore $\left\{p_{n}\right\}$ is Cauchy sequence, more specifically 0 -Cauchy sequence in $X$. By the completeness of $X$ there exists $z \in X$ such that $\left\{p_{n}\right\} \in M(\mathfrak{m}, X, z)$. Since $\left\{p_{n}, p_{n+1}, \ldots\right\} \subset \Delta_{n}$ and $\Delta_{n}$ is closed for each $n \in \mathbb{N}$, using Proposition 3.1 we have $z \in \cap_{n=1}^{\infty} \Delta_{n}$.

Next we prove the uniqueness of $z$. Let $q \in \cap_{n=1}^{\infty} \Delta_{n}$ be another point, then either $m(z, q)>\mathfrak{m}_{z q}$ or $\mathcal{M}_{z q}>\mathfrak{m}_{z q}$. That is $\max \left\{m(z, q)-\mathfrak{m}_{z q}, \mathcal{M}_{z q}-\mathfrak{m}_{z q}\right\}>0$. As $\operatorname{diam}\left(\Delta_{n}\right) \rightarrow 0$, there exists $N_{0} \in \mathbb{N}$ such that

$$
\operatorname{diam}\left(\Delta_{n}\right)<\max \left\{m(z, q)-\mathfrak{m}_{z q}, \mathcal{M}_{z q}-\mathfrak{m}_{z q}\right\} \leq \operatorname{diam}\left(\Delta_{n}\right)
$$

for all $n \geq N_{0}$, a contradiction. Hence $\cap_{n=1}^{\infty} \Delta_{n}=\{z\}$ and this completes the proof of our theorem.

\section{Fixed point results on $m^{*}-$ metric space}

Definition 4.1. Let $(X, \mathfrak{m})$ be an $m^{*}$-metric space and $T: X \rightarrow X$ be a mapping. Then $T$ is said to be $\mathfrak{m}$-contraction if

$$
\mathfrak{m}(T p, T q) \leq k \mathfrak{m}(p, q)
$$

for all $p, q \in X$, where $k \in(0,1)$. 
Definition 4.2. Let $(X, \mathfrak{m})$ be an $m^{*}$-metric space and $T: X \rightarrow X$ be a mapping. Then $T$ is said to be $\mathfrak{m}$-quasi-contraction if

$$
\mathfrak{m}(T p, T q) \leq k \max \{\mathfrak{m}(p, q), \mathfrak{m}(p, T p), \mathfrak{m}(q, T q), \mathfrak{m}(T p, q), \mathfrak{m}(p, T q)\}
$$

for all $p, q \in X$ and for some $k \in(0,1)$.

Now we come to our main fixed point theorems.

Theorem 4.1. Let $(X, \mathfrak{m})$ be a complete $m^{*}$-metric space and $T: X \rightarrow X$ be a mapping such that it satisfies the following conditions:

(1) $T$ is an $\mathfrak{m}$-contraction; $\infty$.

(2) there exists $p_{0} \in X$ such that $\delta\left(\mathfrak{m}, T, p_{0}\right)=\sup \left\{\mathfrak{m}\left(T^{i} p_{0}, T^{j} p_{0}\right): i, j \geq 1\right\}<$

Then $T$ has a unique fixed point in $X$.

Proof. Let us define $\delta\left(\mathfrak{m}, T^{p+1}, p_{0}\right)=\sup \left\{\mathfrak{m}\left(T^{p+i} p_{0}, T^{p+j} p_{0}\right): i, j \geq 1\right\}$ for any $p \geq 0$. Since $T$ satisfies the contractive condition (4.1), we have

$$
\begin{aligned}
\mathfrak{m}\left(T^{p+i} p_{0}, T^{p+j} p_{0}\right) & \leq k \mathfrak{m}\left(T^{p-1+i} p_{0}, T^{p-1+j} p_{0}\right) \\
& \leq k \delta\left(\mathfrak{m}, T^{p}, p_{0}\right)
\end{aligned}
$$

for all $i, j, p \geq 1$. From (4.3) it follows that

$$
\begin{aligned}
\delta\left(\mathfrak{m}, T^{p+1}, p_{0}\right) \leq & k \delta\left(\mathfrak{m}, T^{p}, p_{0}\right) \\
& \ldots \\
\leq & k^{p} \delta\left(\mathfrak{m}, T, p_{0}\right)
\end{aligned}
$$

for all $p \in \mathbb{N}$. As $k \in(0,1)$ we get $\lim _{p \rightarrow \infty} \delta\left(\mathfrak{m}, T^{p+1}, p_{0}\right)=0$. Therefore $\lim _{n, k \rightarrow \infty} \mathfrak{m}\left(p_{n}, p_{k}\right)=$ 0 and $\lim _{n \rightarrow \infty} \mathfrak{m}\left(p_{n}, p_{n}\right)=0$. Thus $\lim _{n, k \rightarrow \infty}\left(\mathfrak{m}\left(p_{n}, p_{k}\right)-\mathfrak{m}_{p_{n} p_{k}}\right)=0$ and $\lim _{n, k \rightarrow \infty}\left(\mathcal{M}_{p_{n} p_{k}}-\right.$ $\left.\mathfrak{m}_{p_{n} p_{k}}\right)=0$. So $\left\{p_{n}\right\}$ is Cauchy sequence in $X$. By the completeness of $X$ we get some $z \in X$ such that $\left\{p_{n}\right\} \in M(\mathfrak{m}, X, z)$ with $\lim _{n \rightarrow \infty}\left(\mathcal{M}_{p_{n} z}-\mathfrak{m}_{p_{n} z}\right)=0$. But $\lim _{n \rightarrow \infty} \mathfrak{m}_{p_{n} z}=$ $\lim _{n \rightarrow \infty}^{n \rightarrow \infty} \min \left\{\mathfrak{m}\left(p_{n}, p_{n}\right), \mathfrak{m}(z, z)\right\}=0$, follows that $\lim _{n \rightarrow \infty} \mathfrak{m}\left(p_{n}, z\right)=0=\lim _{n \rightarrow \infty} \mathcal{M}_{p_{n} z}$. Thus

$$
\mathfrak{m}\left(p_{n+1}, T z\right)=\mathfrak{m}\left(T p_{n}, T z\right) \leq k \mathfrak{m}\left(p_{n}, z\right) \rightarrow 0
$$

as $n \rightarrow \infty$. Hence $\lim _{n \rightarrow \infty}\left(\mathfrak{m}\left(p_{n+1}, T z\right)-\mathfrak{m}_{p_{n+1} T z}\right)=0$. Also $\mathfrak{m}(z, z)=0$ and by the contractive condition (4.1) we get $\mathfrak{m}(T z, T z)=0$.

$$
\mathfrak{m}(z, T z)-\mathfrak{m}_{z T z}=\mathfrak{m}(z, T z) \leq b \limsup _{n \rightarrow \infty}\left(\mathfrak{m}\left(z, p_{n+1}\right)-\mathfrak{m}_{z p_{n+1}}\right)=0 .
$$

Therefore it follows that $z=T z$ and $z$ is a fixed point of $T$ in $X$. 
If $z$ and $w$ are two fixed points of $T$, then we see that

$$
\begin{aligned}
\mathfrak{m}(z, w)=\mathfrak{m}(T z, T w) & \leq k \mathfrak{m}(z, w) \\
\mathfrak{m}(z, z)=\mathfrak{m}(T z, T z) & \leq k \mathfrak{m}(z, z) \\
\mathfrak{m}(w, w)=\mathfrak{m}(T w, T w) & \leq k \mathfrak{m}(w, w)
\end{aligned}
$$

From which it follows that $\mathfrak{m}(z, w)=\mathfrak{m}(z, z)=\mathfrak{m}(w, w)=0$ implies $z=w$ i.e. $T$ has a unique fixed point in $X$.

Theorem 4.2. Let $(X, \mathfrak{m})$ be a complete $m^{*}$-metric space and $T: X \rightarrow X$ be a mapping such that it satisfies the following conditions:

(1) $T$ is an $\mathfrak{m}$-quasi-contraction with $k \in(0,1) \cap\left(0, \frac{1}{b}\right)$;

(2) there exists $p_{0} \in X$ such that $\delta\left(\mathfrak{m}, T, p_{0}\right)=\sup \left\{\mathfrak{m}\left(T^{i} p_{0}, T^{j} p_{0}\right): i, j \geq 1\right\}<$ $\infty$.

Then the Picard iterating sequence $\left\{T^{n} x_{0}\right\}$ converges to some $u \in X$ which is the unique fixed point of $T$ in $X$.

Proof. Similar as in Theorem 4.1 we define $\delta\left(\mathfrak{m}, T^{p+1}, p_{0}\right)=\sup \left\{\mathfrak{m}\left(T^{p+i} p_{0}, T^{p+j} p_{0}\right)\right.$ : $i, j \geq 1\}$ for any $p \geq 0$. Since $T$ satisfies the contractive condition (4.2), we have

$$
\begin{aligned}
\mathfrak{m}\left(T^{p+i} p_{0}, T^{p+j} p_{0}\right) \leq & k \max \left\{\mathfrak{m}\left(T^{p-1+i} p_{0}, T^{p-1+j} p_{0}\right), \mathfrak{m}\left(T^{p-1+i} p_{0}, T^{p+i} p_{0}\right),\right. \\
& \quad \mathfrak{m}\left(T^{p-1+j} p_{0}, T^{p+j} p_{0}\right), \mathfrak{m}\left(T^{p-1+i} p_{0}, T^{p+j} p_{0}\right), \\
& \left.\quad \mathfrak{m}\left(T^{p-1+j} p_{0}, T^{p+i} p_{0}\right)\right\} \\
& \leq k \delta\left(\mathfrak{m}, T^{p}, p_{0}\right)
\end{aligned}
$$

for all $i, j, p \geq 1$. By similar calculation as in Theorem 4.1 we deduce that the Picard iterating sequence $\left\{p_{n}\right\} \in M(\mathfrak{m}, X, u)$ for some $u \in X$ with $\lim _{n \rightarrow \infty}\left(\mathcal{M}_{p_{n} u}-\mathfrak{m}_{p_{n} u}\right)=0$. Therefore we get $\lim _{n \rightarrow \infty} \mathfrak{m}\left(p_{n}, u\right)=0$ and $\mathfrak{m}(u, u)=0$.

Now for any fixed $n \in \mathbb{N}$ we have,

$$
\begin{aligned}
& \mathfrak{m}\left(u, T^{n} p_{0}\right)=\mathfrak{m}\left(u, T^{n} p_{0}\right)-\mathfrak{m}_{u T^{n} p_{0}} \leq b \limsup _{k \rightarrow \infty}\left(\mathfrak{m}\left(T^{n+k} p_{0}, T^{n} p_{0}\right)-\mathfrak{m}_{T^{n+k}} p_{0} T^{n} p_{0}\right) \\
& =\quad b \limsup _{k \rightarrow \infty} \mathfrak{m}\left(T^{n+k} p_{0}, T^{n} p_{0}\right) \\
& \leq \quad b \delta\left(\mathfrak{m}, T^{n}, p_{0}\right) \leq b k^{n-1} \delta\left(\mathfrak{m}, T, p_{0}\right) .
\end{aligned}
$$

Now,

$$
\begin{aligned}
\mathfrak{m}\left(T u, T^{2} p_{0}\right) & \leq k \max \left\{\mathfrak{m}\left(u, T p_{0}\right), \mathfrak{m}(u, T u), \mathfrak{m}\left(T p_{0}, T^{2} p_{0}\right), \mathfrak{m}\left(u, T^{2} p_{0}\right), \mathfrak{m}\left(T p_{0}, T u\right)\right\} \\
(4.9) & \leq k \max \left\{b \delta\left(\mathfrak{m}, T, p_{0}\right), \mathfrak{m}(u, T u), \delta\left(\mathfrak{m}, T, p_{0}\right), b k \delta\left(\mathfrak{m}, T, p_{0}\right), \mathfrak{m}\left(T p_{0}, T u\right)\right\} \\
& =k \max \left\{b \delta\left(\mathfrak{m}, T, p_{0}\right), \mathfrak{m}(u, T u), \delta\left(\mathfrak{m}, T, p_{0}\right), \mathfrak{m}\left(T p_{0}, T u\right)\right\} .
\end{aligned}
$$

Also,

$$
\mathfrak{m}\left(T u, T^{3} p_{0}\right) \leq
$$




$$
\begin{aligned}
& \leq k \max \left\{\mathfrak{m}\left(u, T^{2} p_{0}\right), \mathfrak{m}(u, T u), \mathfrak{m}\left(T^{2} p_{0}, T^{3} p_{0}\right), \mathfrak{m}\left(u, T^{3} p_{0}\right), \mathfrak{m}\left(T^{2} p_{0}, T u\right)\right\} \\
(4.10) & \leq k \max \left\{b k \delta\left(\mathfrak{m}, T, p_{0}\right), \mathfrak{m}(u, T u), \delta\left(\mathfrak{m}, T^{2}, p_{0}\right), b k^{2} \delta\left(\mathfrak{m}, T, p_{0}\right), \mathfrak{m}\left(T^{2} p_{0}, T u\right)\right\} \\
& \leq k \max \left\{b k \delta\left(\mathfrak{m}, T, p_{0}\right), \mathfrak{m}(u, T u), k \delta\left(\mathfrak{m}, T, p_{0}\right), \mathfrak{m}\left(T^{2} p_{0}, T u\right)\right\} \\
& \leq k \max \left\{b k \delta\left(\mathfrak{m}, T, p_{0}\right), \mathfrak{m}(u, T u), k \delta\left(\mathfrak{m}, T, p_{0}\right), k \mathfrak{m}\left(T p_{0}, T u\right)\right\} .
\end{aligned}
$$

Proceeding in a similar way for every $n \geq 1$ we get,

$$
\begin{aligned}
& \mathfrak{m}\left(T u, T^{n+1} p_{0}\right) \leq \\
\leq \quad & \max \left\{b k^{n} \delta\left(\mathfrak{m}, T, p_{0}\right), k \mathfrak{m}(u, T u), k^{n} \delta\left(\mathfrak{m}, T, p_{0}\right), k^{n} \mathfrak{m}\left(T p_{0}, T u\right)\right\}
\end{aligned}
$$

From (4.11) it follows that $\lim \sup \mathfrak{m}\left(T u, T^{n+1} p_{0}\right) \leq k \mathfrak{m}(u, T u)$. Thus we have,

$$
\begin{aligned}
\mathfrak{m}(u, T u)=\mathfrak{m}(u, T u)-\mathfrak{m}_{u T u} & \leq b \limsup _{n \rightarrow \infty}\left(\mathfrak{m}\left(T^{n+1} p_{0}, T u\right)-\mathfrak{m}_{T^{n+1}} p_{0} T u\right) \\
& =b \limsup _{n \rightarrow \infty} \mathfrak{m}\left(T^{n+1} p_{0}, T u\right) \\
& \leq b k \mathfrak{m}(u, T u)
\end{aligned}
$$

From the inequality (4.12) it clear that $\mathfrak{m}(u, T u)=0$. Since $T$ satisfies the contractive condition (4.2), we get $\mathfrak{m}(T u, T u)=0$. Therefore $T u=u$ and $u$ is a fixed point of $T$.

If $w$ is a fixed point of $T$ in $X$, then we get

$$
\begin{aligned}
\mathfrak{m}(u, w)=\mathfrak{m}(T u, T w) & \leq k \max \{\mathfrak{m}(u, w), \mathfrak{m}(u, u), \mathfrak{m}(w, w)\} \\
\mathfrak{m}(u, u)=\mathfrak{m}(T u, T u) & \leq k \mathfrak{m}(u, u) \\
\mathfrak{m}(w, w)=\mathfrak{m}(T w, T w) & \leq k \mathfrak{m}(w, w) .
\end{aligned}
$$

From which it follows that $\mathfrak{m}(u, w)=\mathfrak{m}(u, u)=\mathfrak{m}(w, w)=0$ implies $u=w$ i.e. $u=w$.

Example 4.1. Let $X=\{1,2,3\}$ and we define $\mathfrak{m}: X \times X \rightarrow[0, \infty)$ as $\mathfrak{m}(1,1)=1$, $\mathfrak{m}(2,2)=2, \mathfrak{m}(3,3)=0$ and

Example 4.2. Let $X=\{1,2,3\}$ and we define $\mathfrak{m}: X \times X \rightarrow[0, \infty)$ as $\mathfrak{m}(1,1)=2$, $\mathfrak{m}(2,2)=1, \mathfrak{m}(3,3)=0$ and

Corollary 4.1. The conclusion of Theorem 4.2 can be made also by using the following contractive conditions instead of contractive condition (4.2):

(a) $\mathfrak{m}(T p, T q) \leq \alpha[\mathfrak{m}(p, T p)+\mathfrak{m}(q, T q)], \alpha \in\left(0, \frac{1}{2}\right) ;$

(b) $\mathfrak{m}(T p, T q) \leq \beta[\mathfrak{m}(p, T q)+\mathfrak{m}(q, T p)], \beta \in\left(0, \frac{1}{2}\right)$;

(c) $\mathfrak{m}(T p, T q) \leq \xi[\mathfrak{m}(p, q)+\mathfrak{m}(p, T p)+\mathfrak{m}(q, T q)], \xi \in\left(0, \frac{1}{3}\right)$;

(d) $\mathfrak{m}(T p, T q) \leq \omega[\mathfrak{m}(p, q)+\mathfrak{m}(p, T q)+\mathfrak{m}(T p, q)], \omega \in\left(0, \frac{1}{3}\right)$;

(e) $\mathfrak{m}(T p, T q) \leq p \mathfrak{m}(p, q)+q \mathfrak{m}(p, T p)+r \mathfrak{m}(q, T q)+s \mathfrak{m}(p, T q)+t \mathfrak{m}(T p, q)$, $p, q, r, s, t \in(0,1)$ with $p+q+r+s+t<1$; 
$(f) \mathfrak{m}(T p, T q) \leq \gamma \max \{\mathfrak{m}(p, q), \mathfrak{m}(p, T p), \mathfrak{m}(q, T q)\}, \gamma \in(0,1) ;$

$(g) \mathfrak{m}(T p, T q) \leq \eta \max \{\mathfrak{m}(p, q), \mathfrak{m}(p, T q), \mathfrak{m}(T p, q)\}, \eta \in(0,1) ;$

(h) $\mathfrak{m}(T p, T q) \leq \zeta \max \left\{\mathfrak{m}(p, q), \mathfrak{m}(p, T p), \mathfrak{m}(q, T q), \frac{\mathfrak{m}(p, T q)+\mathfrak{m}(T p, q)}{2}\right\}, \zeta \in$ $(0,1)$.

\section{Application to the stability of fixed point problem}

In this section, we will discuss Hyers-Ulam stability of fixed points of mappings. For more details on Hyers-Ulam stability of functional equations and its applications on fixed point problems one can refer to [4], [11] and [12].

Let $(X, \mathfrak{m})$ be an $m^{*}$-metric space and $T: X \rightarrow X$ be a given mapping. Let us consider the fixed point equation

$$
T p=p, \mathfrak{m}(p, p)=0
$$

and the inequality

$$
\mathfrak{m}(T q, q)-\mathfrak{m}_{T q q}<\epsilon
$$

for any $\epsilon>0$.

Definition 5.1. The fixed point problem (5.1) is said to be Hyers-Ulam stable if there exists an element $c>0$ such that for each $\epsilon>0$ and an $\epsilon$-solution (A solution of (5.2)) $v \in X$ there exists a solution $u \in X$ of the fixed point equation (5.1) such that $\mathfrak{m}(u, v)<c \epsilon$.

Theorem 5.1. Let $(X, \mathfrak{m})$ be a complete $M_{b}$-metric space with coefficient $s \geq 1$ and $T: X \rightarrow X$ be a mapping such that $T$ satisfies all the conditions of Theorem 4.1 with the Lipschitz constant $k \in\left(0, \frac{1}{s}\right)$. Then the fixed point equation of $T$ is Hyers-Ulam stable.

Proof. Since any $M_{b}$-metric space is $m^{*}$-metric space, from Theorem 4.1 we see that $T$ has a unique fixed point $u$ in $X$ with $\mathfrak{m}(u, u)=0$ that is the fixed point equation (5.1) of $T$ has a unique solution. Let $\epsilon>0$ be arbitrary and $v$ be an $\epsilon$-solution of $T$. Then

$$
\begin{aligned}
\mathfrak{m}(u, v) & =\mathfrak{m}(u, v)-\mathfrak{m}_{u v} \\
& \leq s\left[\left(\mathfrak{m}(u, T v)-\mathfrak{m}_{u T v}\right)+\left(\mathfrak{m}(T v, v)-\mathfrak{m}_{T v} v\right)\right] \\
& =s\left[\mathfrak{m}(T u, T v)+\left(\mathfrak{m}(T v, v)-\mathfrak{m}_{T v} v\right)\right] \\
& \leq s\left[k \mathfrak{m}(u, v)+\left(\mathfrak{m}(T v, v)-\mathfrak{m}_{T v v}\right)\right]
\end{aligned}
$$

This implies $\mathfrak{m}(u, v) \leq \frac{s}{1-s k}\left(\mathfrak{m}(T v, v)-\mathfrak{m}_{T v} v\right)<\frac{s}{1-s k} \epsilon$. Therefore the fixed point equation of $T$ is Hyers-Ulam stable. 


\section{Acknowledgements}

The second author acknowledges financial support awarded by the Council of Scientific and Industrial Research, New Delhi, India, through research fellowship for carrying out research work leading to the preparation of this manuscript.

\section{R E F E R E N C E S}

1. S. Matthews: Partial metric topology. Ann. N. Y. Acad. Sci., 728 (1994), 183-197.

2. M. Asadi, E. Karapinar and P. Salimi: New extension of p-metric spaces with some fixed-point results on $M$-metric spaces. Journal of Inequalities and Applications 2014, 2014:18.

3. I. A. Bakhtin: The contraction principle in quasimetric spaces. Func. An., Ulianowsk, Gos. Ped. Ins., 30 (1989), 26-37.

4. S. M. Jung: Hyers-Ulam-Rassias stability of functional equations in nonlinear analysis. Springer Optimization and Its Applications, 48 (2011).

5. M. Jleli, B. Samet: A generalized metric space and related fixed point theorems. Fixed Point Theory Appl. 2015, 14 (2015).

6. M. A. Alghamdi, S. Gulyaz-Ozyurt, E. Karapinar: A Note on Extended ZContraction. Mathematics 2020, 8, 195.

7. E. Karapinar, C. Chifu: Results in wt-Distance over b-Metric Spaces. Mathematics 2020, 8, 220.

8. C. Chifu, E. Karapinar, G. Petrusel: Fixed point results in $\varepsilon$-chainable complete b-metric spaces. Fixed Point Theory, 21(2) (2020), 453-464.

9. A. Fulga, E. Karapinar, G. Petrusel: On Hybrid Contractions in the Context of Quasi-Metric Spaces. Mathematics 2020, 8, 675.

10. N. Mlaiki, A. Zarrad, N. Souayah, A. Mukheimer and T. Abdeljawed: Fixed point theorems in $M_{b}$-metric spaces. Journal of Mathematical Analysis, 7(5) (2016), Pages 1-9.

11. K. Roy and M. Saha: Fixed points of mappings over a locally convex topological vector space and Ulam-Hyers stability of fixed point problems. Novi Sad J. Math., 50(1) (2020), 99-112.

12. K. Roy and M. Saha: On fixed points of Ćirić-type contractive mappings over a $C^{*}$-algebra valued metric space and Hyers-Ulam stability of fixed point problems. J. Adv. Math. Stud., 12(3) (2019), 350-363. 